\title{
The Public Health Community Platform: Implementing Electronic Case Reporting
}

\author{
Marcus Rennick*, Scott Gordon, Monica Huang, Mark Sum and Paula Soper
}

Informatics, Association of State and Territorial Health Officials, Arlington, VA, USA

\section{Objective}

To inform the community on the progress of electronic case reporting (eCR) utilizing the shared infrastructure and applications of the Public Health Community Platform (PHCP).

\section{Introduction}

The PHCP is a community-led initiative to provide shared infrastructure, services, and applications to the public health community as solutions for complex public health informatics problems. The project has progressed by establishing a governance structure led by an executive committee representative of the public health practice community. The executive committee has established the strategic path for the continued development of the PHCP and prioritized eCR as the initial use case for implementation.

Case reporting from clinical providers still requires forms to be manually filled out and sent via mail or fax to the public health agency (PHA). Reporting requirements vary among PHAs and providers may be unaware of reporting requirements and mechanisms in their practicing jurisdiction.

The complexities of case reporting from clinical providers have resulted in inconsistent, incomplete, inaccurate, and delayed reporting of persons that are of interest to public health. This impacts the overall ability of PHAs to interpret surveillance data and take informed action to minimize the population burden of these conditions. The eCR solution coincides with expected Meaningful Use Stage 3 rules that will include case reporting as a public health option.

\section{Methods}

eCR includes the design, development, and implementation of a common electronic infrastructure (i.e., content, platform, and tools) to leverage EHRs so clinical care can more efficiently send standardsbased, secure, and confidential case reports for reportable conditions to state, territorial, local, and tribal PHAs.

There are several components needed to achieve eCR, including:

- Standard data extractions from EHRs

- Data transaction and transportation protocols

- Trigger codes to filter clinical encounters and identify those most likely to include a reportable condition

- Decision support rules and logic defined by each jurisdiction to determine if a filtered patient encounter is reportable in the relevant jurisdiction

- Web forms and forms managers to collect additional data elements for public health

The PHCP is currently coordinating the development, integration, and deployment of these components. This includes the successful completion of an HL7® standard for an initial case message and implementation and integration of a production version Reportable Conditions Knowledge Management System (RCKMS) under development by $\mathrm{CSTE}^{1}$ (integral to providing jurisdictionally defined rules for decision support logic). The information flow is illustrated in Figure 1.

The process is designed for PHA customization due to the variability in state and local reporting requirements. Centrally locating customization on the PHCP relieves the burden on EHR systems to account for all permutations of PHA differences, allowing PHAs to modify their data collection based on emerging needs. Furthermore, PHCP-located resources decreases the need for PHAs to locally create duplicative services and applications to support eCR.

\section{Results}

Pilot teams consiting of PHAs, EHR vendors, and clinical providers are being formed to test this information flow and integration with the PHCP and RCKMS.

\section{Conclusions}

As data collection becomes automated with eCR, it is expected that the PHA workforce will be redirected toward data analysis, interpretation, response, and prevention (rather than focusing on the completion of case report forms). As that data is transformed into knowledge, public health will be able to provide greater input into the Learning Health System and improve health outcomes in the population.

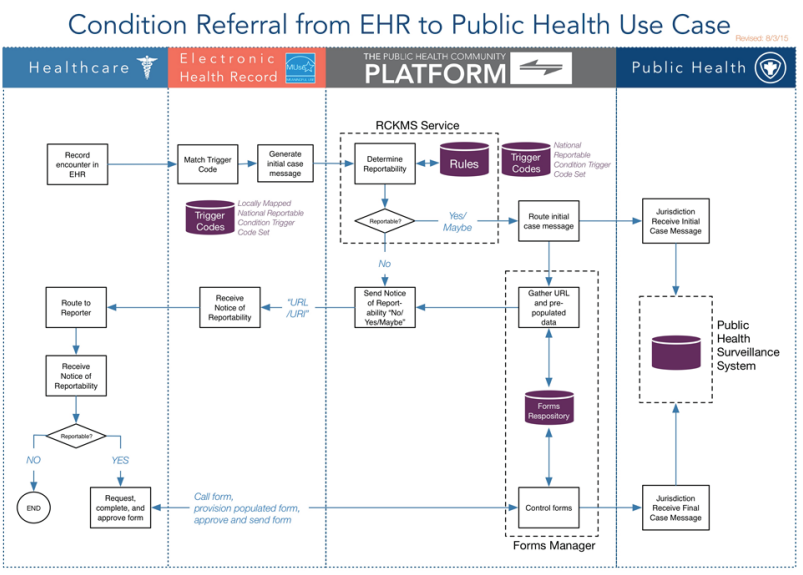

Figure 1. Transactional information flow for eCR.

\section{Keywords}

eCR; case reporting; Public Health Community Platform; interoperability

\section{Acknowledgments}

The PHCP Executive Committee: Art Davidson (NACCHO co-chair), J.T. Lane (ASTHO co-chair), Bill Brand, Jim Collins, Mark Conde, Rebecca Coyle, \& Bryant Karras.

CDC/CSELS

\section{References}

1. Council for State and Territorial Epidemiologists. Surveillance Informatics: Reportable Condition Knowledge Management System [Internet]. Atlanta (GA): CSTE; No Date [cited 2015 Aug 31]. Available from: http://www.cste.org/group/RCKMS.

\section{*Marcus Rennick}

E-mail: mrennick@astho.org 